\title{
Le problematiche della famiglia borghese nel Secondo dopoguerra in Una spirale di nebbia e ne I cieli della sera di Michele Prisco
}

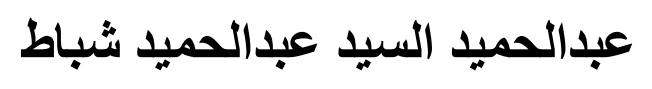

La famiglia si considera il nucleo principale di tutta la società e il centro di aggregazione sentimentale. Inoltre, essa è il primo nucleo di compagine sociale. Prisco tratta la famiglia, soprattutto quella del Sud, caratterizzata da una sorta d'imprigionazione dell'individuo in quanto il Meridione è un ambiente chiuso con tradizioni e costumi molto radicati. Dato che la famiglia rispecchia la società con tutti i suoi valori. Prisco analizza in dettaglio alcune delle crisi che riscontrano la famiglia e soprattutto nel Secondo dopoguerra.

La famiglia, nel Secondo dopoguerra, ha dimostrato varie crisi, soprattutto le mortificazioni, le ambizioni, le afflizioni e gli egoismi, segno della crisi della società. Prisco tratta il tema della famiglia da diversi lati nella sua narrativa: le relazioni coniugali, i problemi della convivenza e la questione del divorzio fra gli sposi come in Una spirale di nebbia da un lato e da un altro, le relazioni fra $\mathrm{i}$ membri della famiglia, l'effetto della mancanza dei genitori nella vita dei figli, la vendetta come tradizione seguita soprattutto nell'ambito delle famiglie del Sud ed i suoi effetti negativi sulla famiglia e sulla società intera ne I cieli della sera. Prisco discute in tutt'e due romanzi questi aspetti che prevalgono nell'ambito della famiglia nel Secondo dopoguerra. Egli dimostra quanti disagi ha riscontrato la famiglia in tal tempo e come la famiglia, soprattutto quella borghese, si è trasformata, attraverso questi disagi. Il trattamento del tema della famiglia indica quanto lo scrittore s'interessi a dare una particolare attenzione alle tradizioni della sua società tramite l'analisi della famiglia, come indica Carlo Bo:

"Prisco a un certo punto della sua carriera abbia preferito fare marcia indietro e navigare sempre di più verso l'isola della tradizione". ${ }^{i}$

Dato che la famiglia è il luogo in cui si possono verificare diversi conflitti con i suoi membri, come l'interesse, le umiliazioni sociali, i conflitti di classe e 
d'ambizione, Prisco sceglie, soprattutto, l'ambito della famiglia per indagarlo. Prisco tratta i conflitti familiari, riferendo che il trattamento di questi conflitti aiuta a trovare una soluzione a questi problemi, per una società perfetta senza crisi né problemi. ${ }^{\text {ii }}$

Altri problemi e vizi della famiglia, specialmente quella meridionale, vengono trattati come la fuga dalle abitudini e dalle mentalità, Prisco difatti intende avvisare la società di questi disagi tramite il trattamento del tema della famiglia nella sua narrativa, cercando di indagare tutto quello che circonda la famiglia al fine di comprendere la realtà della società intorno come Martin Gistucci indica:

"Mettendo l'inferno del familiare al centro dei suoi romanzi, Prisco ha inteso conferirgli una dimensione simbolica. La famiglia è rappresentata in modo emblematico tutta la società".iii

Il concetto della famiglia potente è evidente in Una spirale di nebbiaquando lo scrittore indica quanto potente sia il clan Sangermano che fa di tutto per salvare Fabrizio dato che porta il loro cognome.

"D'altronde attraverso questo sondaggio iniziale bisognava pure studiare il comportamento da assumere e non tanto magari nei confronti di Fabrizio, il quale dopo tutto portava il loro cognome e aveva diritto all'appoggio della famiglia colpevole o innocente che sia [...]. Solo che per il momento nessuno osava affrontare l'argomento. Ci sono certe regole da rispettare, nella famiglia, e la consuetudine è che a porre per il primo le questioni sul tappeto sia suo nonno Pietro Sangermano, il grande vecchio, ".iv

Prisco dà importanza all'analisi etico-psicologica giacché essa è il punto di riferimento sentimentale; egli esamina, nella sua scrittura, questi sentimenti fra i membri della famiglia sia quando in questa famiglia è riferente a una coppia coniugale come Una spirale di nebbia sia quando essa è riferente a quelli dei genitori e i figli come ne I cieli della sera. Il mondo è cambiato nel Secondo dopoguerra, avendo tante problematiche come la decadenza morale e non seguendo 
più i valori tradizionali. In Una spirale di nebbia, Prisco tratta un vero clan, cosciente del proprio prestigio, geloso delle sue prerogative, pronto a tutto pur di salvare la faccia e serbare un posto di rango. In questa famiglia, il patriarca, il "gran vecchio", veglia sulla salvaguardia dell'immagine irreprensibile della sua comunità familiare, soltanto per "ragione di stato" e per il pensiero dell'intangibile rispettabilità della famiglia. Egli si sforza di salvare Fabrizio,l'accusato di uccidere sua moglie, mentre cacciavano nella foresta. La famiglia di Sangermano è grande e numerosa con una forza che non sa assurdità come indica Prisco:

"Il ragionamento materno non si potesse dire proprio assurdo: perché in un paese così incline alla forza della raccomandazione, e così lesto a imboccare la via dei compromessi $[\ldots]$. Naturalmente si era trattato d'una riunione preliminare e neppure ormai e suddivisa in parecchi rami in linea collaterale e discendente". ${ }^{\mathrm{v}}$

Gli interessi, le umiliazioni sociali, i conflitti di classe, d'età, d'ambizione, le disparità di prestigio e di fortuna fanno della famiglia una metafora della società imperfetta che la attornia. L'individuo, alienato nella cellula familiare, si fissa in uno stadio infantile che non supera mai, come nel caso di Fabrizio Sangermano, che s'interessa della collezione di farfalle! L'alienazione può anche prendere la forma d'un disordine psichico: è il caso di Marcello, figlio docile delle donne che hanno governato la sua infanzia e diventato impotente, incapace di assumere il suo ruolo di maschio. ${ }^{\mathrm{vi}}$

"Ma di là di questa disparità di comportamento tutta esteriore c'era qualcosa che non era più, sì, né la pigrizia incoraggiata dal suo ambiente familiare nè la sorridente socievolezza o la programmatica austerità della sua vita pubblica ma era piuttosto qualcosa di più sotterraneo e inalienabile che s'aggiungeva ogni volta ad uno di questi diversi atteggiamenti, per cui, avrebbe potuto riconoscerlo sempre e dirsi è lui, è Marcello, non cambia mai, non cambierà mai". vii 
La famiglia è una catena che vale tanto per la prigionia quanto per la solidarietà; rompere la catena è il solo mezzo per evaderne, ma la rottura non avviene senza dolore sia per l'individuo sia per il resto della famiglia. La famiglia è un insieme d'individui legati da vincoli di matrimonio, parentela, affinità, adozione, tutela, o da vincoli affettivi che dimorano abitualmente sotto lo stesso tetto. Questa definizione è un punto di partenza per individuare la popolazione di riferimento negli studi che disegnano un quadro preciso della famiglia. ${ }^{\text {vii }}$

In Una spirale di nebbia, Prisco tratta le relazioni coniugali e il conflitto sociologico, indicando che a causa delle incomprensioni e delle delusioni, degli errori, delle ipocrisie e delle differenze dei ceti sociali a cui attribuiscono gli sposi nascono questi problemi. Tutto questo spezza in tal modo il vincolo matrimoniale e come conseguenza ne risulta l'insoddisfazione reciproca che alla fine conduce a interrompere ogni comunicazione matrimoniale.

I Sangermano non volevano neanche vedere Valeria in quanto non avevano voglia di avere elementi nuovi nella loro famiglia e preferivano il matrimonio di cugine, per mantenere così la famiglia compatta. Valeria è settentrionale perciò il suo accento è più stretto e marcato, e il fatto diventa un motivo di riprovazione. Oltre a ciò, lei rappresenta un ceto inferiore di quello dei Sangermano perchéè figlia di un ragioniere che, secondo loro, è il simbolo della mediocrità milanese più sfacciata. ${ }^{\text {ix }}$

Il clan Sangermano, con tutte le sue diramazioni e con tutti gli intrighi che lo denotano, è una cellula qualsiasi dell'organismo sociale che solo per caso si colloca in un punto specifico del cosmo. Il padre assume il suo ruolo, ed è il tipo di padre dominante, come lo descrive Prisco:

"Suo padre aveva scelto, o subito, d'essere un capo tribù? Ma svolgeva quel compito senza farlo pesare come un grosso baco da seta che inghiotte il disordine quotidiano e lo fila e restituisce un filo lucido e forte pronto per tessere". ${ }^{\mathrm{x}}$ 
Volendo indagare i sentimenti umani, Prisco si accorge che il migliore campo per studiare le relazioni dei sentimenti umani è l'universo provinciale poiché è un ottimo campo d'osservazione e il centro dei sentimenti umani che si trova particolarmente nell'indole familiare. InUna spirale di nebbia, Prisco discute uno dei problemi più urgenti nella sua società, soprattutto nel Secondo dopoguerra, iniziando con la coppia di Fabrizio e Valeria, confrontando i due sposi nella loro vita, mettendo in rassegna altre coppie da indagare per arrivare a dimostrare quanti problemi reali ci siano fra una coppia che vive sotto lo stesso tetto. ${ }^{\mathrm{xi}}$

Lo scopo principale di Prisco, secondo Lorenzo Sbragi, è di intendere la commedia umana nelle sue ragioni perciò la sua analisi dipende sempre dall'uomo. Così,quest'analisi della condizione matrimoniale mira a recuperare l'immagine dell'uomo. Per questo motivo i casi, che Prisco ricorda, sono relativi all'indole familiare, e rientrano tutti nel chiuso delle pareti domestiche che includono conflitti e congiure coniugali. . $^{\mathrm{ii}}$

Egli tratta così la "famiglia - prigione" per l'individuo, discutendo l'aspetto del "matrimonio all'italiana" che si considera uno dei problemi più scottanti della società italiana. Prisco affronta inoltre, nella sua narrativa, la questione del divorzio. Lo scrittore mette in risalto una densa tessitura drammatica di tante situazioni d'incompatibilità coniugale, dimostrando che Fabrizio non è felice nella sua vita matrimoniale per i problemi dell'inettitudine: il suo matrimonio è pieno di problematiche e di disagi, oltre alle altre coppie coniugali che rappresentano altri diversi tipi di problemi legati alla famiglia e come conseguenza a tutta la società. ${ }^{\text {xiii }}$

Prisco tratta in Una spirale di nebbia la mancanza della madre di Fabrizio,dovuta alla reclusione in una casa di cura; tale mancanza ha provocato un effetto negativo su di lui e in particolare quando lei non lo riconosce più a causa di traumi psicologici come Fabrizio indica:

"Ma è veramente la mamma? Non mi riconosce, non ha capito chi sono! E scoppiò a piangere". ${ }^{\text {iv }}$ 
La situazione prevede altre coppie che convivono e hanno tanti problemi fra amore e abitudine, desiderio e noia, passione e repulsione, interferenze e incomunicabilità. ${ }^{\mathrm{xv}}$ Si ramifica dalla disgrazia dei due sposi Fabrizio e Valeria, altre coppie che ci ripensano sulla loro vita insieme e sui disguidi che anche loro hanno nella loro vita. Fra queste coppie, c'è quella di Renato Marino che vuole respingere la solitudine che si accampa in lui con la complicità del silenzio. Renato Marino ritiene che la solitudine in un matrimonio sia quasi come un delitto. Oltre alla sofferenza della solitudine fra una coppia per motivi di lavoro che rendono i loro incontri difficili, per la rara presenza del marito, ci sono dei problemi causati dalle suocere, come indica Renato Marino parlando di sua madre con la moglie:

"Io, vedi, Lidia, io mi sento in colpa con te, è la verità perché ho accettato l'imposizione di mia madre e non ho saputo importi a lei permettendo che lei ti offendesse ogni giorno per tutti i giorni dell'anno e per tutti gli anni del nostro matrimonio". ${ }^{x v i}$

Prisco mette in rassegna il conflitto tra amore materno ed amore coniugale mettendo tutto in chiave psicoanalitica come indica Prisco nel discorso di Renato Marino di sua madre:

"Senti, mamma, Lidia è mia moglie, e non un'amante o una serva: tu devi convincertene, devi conoscerla. Stasera tela porto a casae la riceverai: non ti chiedo di volerle bene, ti chiedo di salvare le forme, di rispettare la sua dignità; di accettare la vita, insomma". xvii

Questo conflitto è un trauma che condiziona quasi la scrittura di Prisco. Egli tenta di sciogliere questo nodo ambiguo e tormentoso che lega e contamina, fino al delitto, infanzia e virilità, maschismo e sadismo, inoltre conversazione e rivoluzione. ${ }^{\text {xviii }}$

Alla fine, Renato Marino rinuncia al tentativo di imporre alla madre l'accettazione della moglie. Si ricorda del giorno dell'atomica su Hiroshima che la madre l'ha accolto come una furia, lanciando insulti all'indirizzo del marito che proprio era scappato da casa. La madre descrive quante sofferenze aveva in quel 
periodo in cui sentiva le angosce per i fatti del mondo, diventando quasi come una specie di piacere solitario da nascondere e di cui a momenti vergognarsi come la madre di Renato descrive la sua sofferenza:

"La notizia che aveva sconvolto la madre, il fatto bruto, la fuga di suo padre: che quella mattina aveva tagliato la corda lasciandole un biglietto. $\mathrm{E}$ così in quel periodo davanti all'illimitata irragionevole sofferenza materna la sua angoscia per i fatti del mondo fa quasi come una specie di piacere solitario da nascondere e di cui a momenti vergognarsi, e persino con Lidia aveva avuto a un tratto ritegno di parlare liberamente di sé e del padre che li aveva abbandonati e della madre che urlava vendetta, perché gli sembrava tutto troppo meschino egoistico assurdo [...]. E se dopo vent'anni per lei Hiroshima era ancora questo, solo questo: l'egoismo di sentirsi abbandonata, il rancore di registrare una sconfitta". ${ }^{\mathrm{xix}}$

Lo scrittore ricorda che questi disguidi che riscontrano una coppia coniugale hanno un effetto negativo sulla loro relazione poichè causa la frustrazione, l'ozio, la noia, l'alienazione, e la privazione della libertà personale. Lo scrittore mira, con la discussione delle diverse ragioni di varie coppie, a conoscere i motivi delle problematiche fra queste coppie, escludendo la banale idea della felicità domestica.

Attraverso la rassegna di altre coppie che vivono sotto lo stesso tetto, Prisco dimostra quanta falsità, alienazione, inganni e fallimenti di follia e di dubbiezza dispiegano il processo di disgregazione del rapporto coniugale insieme alla correlata dissacrazione dell'istituto familiare sotto la spinta dell'intollerabilità di ogni legame che la civiltà del benessere ha modificato. ${ }^{\mathrm{xx}}$

Prisco tratta la coppia di Marcello Testa e Maria Teresa. Marcello si è sposato con Maria Teresa, nascondendole il fatto che era completamente impotente:

"Solo che a vederlo in quella circostanza diventava quasi difficile immaginare che fosse lo stesso uomo il quale a sera, a casa, quando si ritiravano in camera, almeno prima di dividere le loro camere, e veniva 
ormai il momento di mettersi a letto, cominciava ad avere nello sguardo quell'espressione torbida sperduta che riusciva comunque abbastanza bene a mascherare la disperazione o magari il terrore $[\ldots] "$. xxi

Dato che questi inganni sono dei motivi che causano i conflitti nella vita famigliare, Prisco dimostra che si tratta di una singolare esperienza che ha condotto al desiderio della moglie di annullare il matrimonio per la mancata consumazione. Quando Marcello scopre la voglia della moglie, per salvare la sua reputazione, escogita di riconoscere come padre il bambino dell'autista Alfredo che ha fatto fare ad Armida, la domestica.

La casa dell'infanzia è prima di tutto un"rifugio": soprattutto la camera dei ragazzi. L'adulto aspira a ritrovarla. Prisco focalizza l'amore materno che Marcello subisce, appena arriva da sua madre, una trasformazione che equivale a un'autentica regressione all'infanzia. La madre e le sorelle lottavano per riconquistare il possesso, regalandogli delle orribili cravatte. .xii $^{\text {. }}$

Prisco accenna che la madre di Marcello Testa respinge il marito per meglio consacrarsi al figlio giacché si sente rivalutare dal figlio maschio; così si spiega l'assimilazione frequente nella madre fra l'amore e l'esecuzione dei compiti materiali come indica lo scrittore.

"Ma allora, io non ti basto più? Non sei più contento di me? Eppure ti ho servito come un prete all'altare. I migliori bocconi sono stati per te, le camicie sempre pronte e stirate nei cassetti, di chi puoi lagnarti? Dimmelo o non mi vuoi più bene?". xxiii

Dato che il padre di Marcello Testa è dominatore e irreprensibile, Prisco mette in risalto l'influenza dell'assenza del ruolo del padre, indicando che il figlio è rimasto sottomesso alla madre. Questo è il caso di Marcello Testa che appena rimette i piedi nella casa materna, è circondato da tre sorelle che sono il prolungamento della madre, gran sacerdotessa del culto del figlio maschio.

C'è stata l'infermiera Lavinia, amante del medico fascista Vittorio Conte che per anni raccontava delle storie false di un matrimonio inesistente che riguarda dei 
famigliari morti in un bombardamento, mentre si trovava a combattere in Sicilia. Per verificare le sue storie, lei si reca qualche volta a Napoli, dove c'è la clinica del professore Botta dove lavora Vittorio, il suo allievo. Il professore Botta è vecchio e si è sposato tardi con una donna molto giovane. All'istituto della clinica;si conosce come un uomo privo d'ideali e si sussurra che sia amante della giovane Botta. Oltre a tutto questo, è ambizioso di fare carriera ingombrando la scrivania di libri e riviste mediche. ${ }^{\text {xiv }}$ Quindi, Prisco indica com'è ridotta la moralità sulle esemplificazioni ambientali ritratte dalla sua società per dare diversi esempi dalla cronaca quotidiana.

Ne I cieli della sera, appena torna Davide alla sua stanza d'infanzia,ricorda tutti i giorni passati nella casa familiare:

"Ero di nuovo nella mia camera d'un tempo: con quel parato a fiorellini bianchi e azzurri che ripeteva l'identico disegno della tenda ai balconi e della coperta sopra il letto, non quei mobili dal leggero misterioso profumo del legno vecchio così simile all'odore delle sacrestie di campagna o delle rose disseccate, e con quel caminetto d'angolo che ricordavo sempre spento e dove da bambino ero andato tante volte a rincantucciarmi come all'interno d'una nicchia per sfogare con le lacrime un torto patito o un capriccio camuffato da torto: ma che cosa avevo in comune, io, con il bambino che aveva abitato questa stanza?". xxv

Prisco tratta qui la famiglia da un lato nuovo, quello dei genitori e dei figli, e come la mancanza dei genitori fa cambiare la vita di tutta la famiglia. Si tratta di una tradizione della vendetta atavica poiché i figli vogliono vendicarsi per la morte dei genitori. Tutta la trama si basa sulla discussione bilanciata fra Giustina che segue la tradizione atavica della vendetta e suo fratello Davide che invita per la tolleranza.

Prisco rappresenta, ne I cieli della sera, il conflitto fra i fratelli che hanno perso i loro genitori in condizioni misteriose, di come reagiscono verso la vendetta di quelli che hanno causato la loro morte. Questo conflitto è stato raffigurato tra Davide e sua sorella Giustina. In questo modo, lo scrittore considera la famiglia 
come un teatro tramite cui rappresenta alcune delle tradizioni e dei vizi della sua società, come la rivalsa, iniziando dalla famiglia, dato che essa è il nucleo riferente per tutta la società. Giustina dimostra come la casa della famiglia ha ancora dei ricordi di tutto quello che si è vissuto e raccontato e ingabbia anche il futuro fra le sue pareti. ${ }^{\mathrm{xx} \mathrm{i}}$

Prisco tratta una delle tradizioni della famiglia che riguarda la vendetta, il sangue e come il passato non si cancelli dato che si tramanda da una genrazione all'altra. Come conseguenza, tutto questo conduce alla delusione e alla disperazione con il sentimento della sconfitta non solo per l'individuo ma per tutta la famiglia come indica Davide e per questo egli è interessato a sapere la verità di questa vicenda che ha causato la malinconia per la sua intera famiglia:

"Mi guardavo attorno un pò deluso, quasi aspettando che accadesse qualcosa a rompere il silenzio: non so cosa, anche un grido del sangue per esempio, come una sorta di premonizione, ad avvertirmi che il passato non era cancellato e che antiche mute terribili ore di quegli anni lontani sarebbero tornate ben presto a riaffollarmi attorno con la paziente e un pò vendicativa sicurezza di chi ha saputo attendere il momento estremo e improrogabile della verità". xxvii

L'assenza del padre è sentita da Davide un'offesa personale. Quest'assenzasi trasforma in ostilità per Davide. Prisco descrive come Davide recupera il suo affetto per il padre e raggiunge la tranquillità che era chiaro nei suoi discorsi con Giustina come le indica"in ogni caso a ricostruire fra di noi, subito, l'intimità interrotta dalla sua assenza. E se quello era stato, e così era stato, il mio rapporto d'affetto con mia madre, se l'avevo amata, e così l'avevo amata, con tanta appassionata confidenza, come riuscito proprio io - o potevo esserne capace - d'averne offeso la memoria, dentro di me, identificando, e accettando, quel preciso movente all'operato di nostro padre?".xxviii 
Davide cerca di recuperare il rapporto figlio-padre; per arrivarci occorre che egli si liberi del ricordo mitizzato della madre, che ne compia "l'omicidio" simbolico.

"Sì, adesso mene sarei partito definitivamente - diventava l'unica maniera di liberarmi da questa specie di malia, l'unico modo d'invocare gli esorcismi- ma nello stesso tempo sarei partito per sempre privo di ogni sostegno interiore. Non voglio dire che avrei adesso cancellato il ricordo di mia madre, dentro di me, voglio dire solo che l'avrei lasciato qui nella sua casa, perché fra noi, fra la mia devozione e la sua irrequietezza, si frapponeva ormai quest'ombra ambigua". xxix

Giustina conferma a Davide che Marta invidiava sua madre, cercando di convincerlo della vendetta per la morte dei loro genitori:

"Perché Marta per la mamma ormai non era più una donna, era il male: il male di cui le creature umane non sono responsabili, che viene da fuori, penetra nell'uomo e ne fa il suo schiavo. Tu credi sempre in Dio, Davide? E allora se credi in Dio, devi credere anche nel male, in questa perpetua lotta fra il bene e il male". xxx

Dato che la madre ha un effetto molto importante nell'ambito della famiglia, lei conta sempre sia presente sia assente, anzi lei conta di più quando è assente, Prisco tratta l'effetto dell'assenza della madre ne I cieli della sera, poiché è morta in un incidente in condizioni misteriose. Egli dimostra quanto è importante la madre per tutta la famiglia soprattutto per Giustina che la menziona sempre nel suo discorso.

Prisco s'impegna in una presa di coscienza, d'approdo di una verità e di una moralità che Davide cerca di diffondere, insieme tolleranza, nella famiglia, contro la violenza e l'odio che sono rappresentati da sua sorella Giustina. Egli cerca di diffondere un senso di razionalità nella famiglia come centro di tutta la famiglia, perciò Prisco si approda ad una verità e, soprattutto, ad una moralità, spinto da un'esigenza di chiarezza verso il mondo stesso. ${ }^{\mathrm{xxi}}$ 
L'importanza di trattare la famiglia e di indagarla in vari modelli storici in cui s'incarna nelle diverse classi sociali. La famiglia rappresenta una parte della società e si combina con un certo sistema sociale, un sistema culturale che incorpora i modelli di comportamento concernente i sistemi sociali. C'è un sistema ecologico che riguarda principalmente la struttura socio-demografica della popolazione e della distribuzione di ulteriore relazione ai problemi di adattamento all'ambiente. La società italiana si configura in una specifica formazione sociale con relativa struttura a due forme dominanti, quella premoderna o (tradizionale) e quella capitalistica e fra loro in rapporto di dominanza (subordinazione) dinamicamente struttura lungo il percorso storico. ${ }^{\text {xxxii }}$

Anche l'assenza del padre è sentita nel discorso di Davide ne I cieli della sera, perché Davide recupera l'affetto per suo padre nelle conversazioni con Giustina in quanto dice forse sempre "nostro padre", mentre Giustina indica alla madre quasi sempre con "nostra madre" per indicare la nostalgia per tutta la famiglia di sua madre.Tutta la storia di Davide gira attorno a questo recupero del rapporto figliopadre e nello stesso momento egli si libera al ricordo mitizzato della madre, chiedendo a Giustina di liberarsi anche lei all'ossessione dell'uccisione o della rivalsa. ${ }^{x x x i i i}$

Ne I cieli della sera, la famiglia è già una fonte di soffocamento in quanto tutto il dramma nasce dalla mancanza dei due genitori. Questa mancanza aggrava tragicamente il vizio fondamentale di ogni struttura familiare. In questo senso, Prisco ha un modo particolare per tessere le sue opere con un filo che lega tutti i libri, come indica Carlo Bo:

"Si provi a confrontare i suoi libri gli uni con gli altri ma sempre in ordine di tempo e si vedrà che c'è in lui e ben visibile una linea continua, un filo che lega i vari momenti dell'ispirazione e nello stesso tempo un rinnovarsi costante e sempre oculato e controllato".xxiv

C'è anche, ne I cieli della sera,la mancanza di tutti i due genitori che rappresentano un'influenza negativa su tutta la famiglia e sui suoi figli nella loro 
vita. Prisco infine cerca di capire l'uomo ed i suoi sentimenti. Egli evoca reazioni e vicende riconducibili a condizioni umane che non si legano con la ristrettezza geografica. Prisco indica inoltre che nella casa-famiglia sulle pareti, tutto è registrato.

"In una vecchia casa tutto quello che si è vissuto e raccontato un tempo resta ingabbiato fra le pareti in attesa come dire? Che il futuro lo ascolti". ${ }^{x x v}$

Prisco mira a dare, attraverso il trattamento delle famiglie sia dei genitori e figli che delle coppie coniugali, una prospettiva generale per tutta la società. Egli cerca nella condizione umana e nelle sue implicazioni socio-ambientali, affondando nell'anima dell'uomo a partire dal matrimonio. ${ }^{\mathrm{xxxvi}} \mathrm{Con}$ il modo di cercare dentro l'uomo, Prisco rievoca episodi e consuetudini di vita, inoltre il discorso e la riflessione si appuntano sull'interiorità degli uomini e degli eventi per il motivo di coglierne il valore più vero, perciò si può parlare estesamente di un mondo e di un universo provinciale.

Il risveglio, la fuga dalle abitudini e dalle mentalità sorpassate passa per quell'italiano del Sud che è Michele Prisco, attraverso la necessità di prendere le distanze dall'universo canonizzato della famiglia; cellula di base di questa società che bisognerebbe ben rimettere in discussione. ${ }^{x x x v i i}$ Prisco inizia dal centro nucleare della società che è la famiglia, intendendo di diffondere la tolleranza come fenomeno in tutta la società, questo è l'impegno morale dello scrittore. Egli mira al trattamento del tema della famiglia. Oltre a questo, s'impegna a fare attenzione a togliere questi mali dalla società tramite il trattamento dei fenomeni negativi sparsi nella società nel Secondo dopoguerra, rappresentando questi fenomeni nell'ambito famigliare poiché rispecchia tutta la società alla fin fine. Così e come conseguenza, risulta che la società si allontani dal commettere questi errori gravi che conducono alla fine alla distruzione di tutta la stessa. 
${ }^{\mathrm{i}}$ Carlo Bo, In introduzione di La dama a piazza di Michele Prisco, Rizzoli, Milano 1961, p. iii.

${ }^{i i}$ Cfr., Martin Gistucci, Michele Prisco e l'inferno da L'Osservatore politico-letterario, n. 2, 1979, p. 6.

iiiIvi., op.cit., p. 4.

${ }^{i v}$ Michele Prisco, Una spirale di nebbia, Rizzoli, Milano, p. 30.

'Ivi., op.cit., p. 29

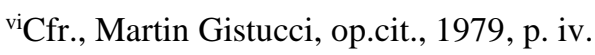

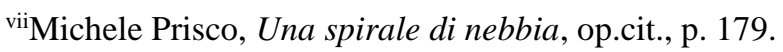

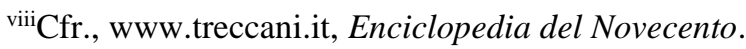

${ }^{\mathrm{ix}}$ Cfr., Michele Prisco, Una spirale di nebbia, op.cit., p. 152.

IIvi., p. 149.

${ }^{x i}$ Cfr., Luciano Luisi, Una vita per la cultura, Ente Fiuggi, Fiuggi 1985, pp. 79-80.

xiiLorenzo Sbragi, Una spirale di nebbia da Il mattino del 26 maggio 1966.

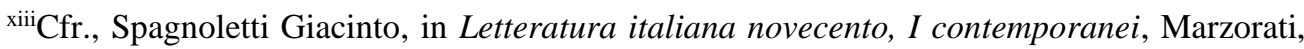
Milano 1979, p. iii.

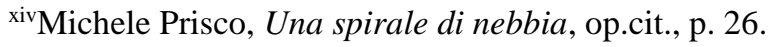

${ }^{\mathrm{x}} \mathrm{Cfr}$., ivi., p. iv.

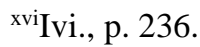

xviiIvi., p. 254.

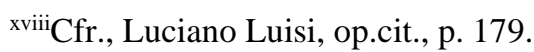

xix Ivi., p. 258.

${ }^{\mathrm{x}}$ Cfr., Carmelo Aliberti, La narrativa di Michele Prisco, Pastogi S.R.L, 31 dicembre 1996, p. 26.

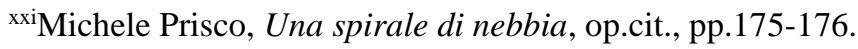

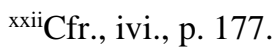

xxiii Ivi., p. 239.

${ }^{\text {xxiv }}$ Cfr., ivi., p. 145

${ }^{\mathrm{xxv}}$ Id., I cieli della sera, op.cit., p. 17.

${ }^{\mathrm{xxvi} C f r}$., ivi., p. 27.

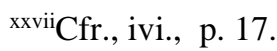

xxviii Ivi., p. 55.

${ }^{x x i x}$ Ivi., p. 226.

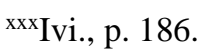


${ }^{\mathrm{xxxi} C f r}$., Carmelo Aliberti, op.cit., p. 60.

${ }^{x x x i i}$ Achile Ardigo, Classi sociali e strati nel mutamento culturale, editrice la scuola, Brescia 1947, p. 200.

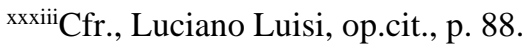

${ }^{\mathrm{xxxiv}}$ Carlo Bo, In introduzione di La dama a piazza di Michele Prisco, op.cit., p. iii.

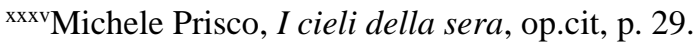

${ }^{x x x v i}$ Cfr., Andrea Di consoli, In Introduzione di Una spirale di nebbia di Michele Prisco, op.cit, p. xiii.

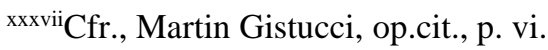

